\title{
Network externalities and Universal Service Obligation in the Internet ${ }^{1}$
}

\author{
Jacques Crémer \\ IDEI-GREMAQ \\ Université de Toulouse 1
}

September 1999

${ }^{1}$ This paper was presented on 3 September 1999 at the European Economic Society meetings in Santiago de Compostella. The ideas discussed here were first presented at the conference on the Universal Service Obligation held at the London Business School on 29 April 1999. Thanks to Len Waverman for forcing me to think about these issues as well as to the participants at the conference and to Claude Crampes and Robin Mason for very useful comments. Thanks also to Mary Kay Perkins whose paper (Perkins, 1999) provided inspiration. 


\section{Introduction}

In recent months, we have witnessed much debate about the desirability of some type of Universal Service requirement for the Internet. The literature has identified four main reasons ${ }^{1}$ for imposing universal service requirements: income redistribution, the presence of "merit goods", regional policy and network externalities.

The aim of this paper is to present a simple model of network externalities when two technologies are in competition for broadcasting information, and to show that there are reasons to believe that the value of the network is increasing fast when one reaches close to $100 \%$ connection. This might provide the basis for a policy of universal access.

The basic idea of the theory is very simple. In a network such as the Internet, there must be decreasing returns to scale in the provision of access to a greater proportion of the population: as more and more persons are connected, the network reaches consumers who attach smaller and smaller value to the services of the network. ${ }^{2}$ However, once we get close to universal access, there are new services which can be offered, or rather there exist new opportunities to deliver what I will call broadcasting services. Take the example of a school that needs to send grades at the home of its pupils. It normally sends them through "snail mail". If enough households were connected to the Internet, it would be beneficial to send it by E-mail, as one saves on postage and gains in speed and flexibility. It is easy to imagine a number of such examples, telephone and electricity bills being one of them (according to Gates (1999), the US Commerce Department estimates that when consumers are able to pay bills on line, annual processing costs will drop by $\$ 20$ billion). The aim of section 2 is to build a simple model that shows that under these circumstances, the social value of the network as a function of the number of participants can be concave for small numbers of participants, but have a sharply increasing section for larger numbers. (Perkins (1999) studies the use of electronic mail to fulfill the postal USO.)

In section 3, I review the model in order to show that these network externalities can create coordination failures, even if the senders of information

\footnotetext{
${ }^{1}$ Each of these reasons could be the topic of a long discussion. The fact that they are listed does not necessarily imply that I agree that the policies based on these reasons are sound.

${ }^{2}$ There is a counterbalancing effect: as the size of the network increases, an additional member provides the service of being accessible to more and more people. This is the basis of Metcalfe's law that states that the value of a network is approximately equal to the square of the number of members. In reality, for each member the value of an extra potential connection also decreases with the size of the network, and I believe that in most cases, once the network has reached a critical size, the statement of the text if valid.
} 
maximize social welfare. I discuss the techniques that the government could use to mitigate the resulting inefficiencies, and show that when there are too few connections to the network, it is more efficient to subsidize the senders of messages than the recipients.

\section{The shape of network externalities}

\subsection{The model}

We build a simple model in which there are two techniques for "broadcasting" information, a postal service and the Internet, and we compute the social welfare generated by this broadcasting as a function of the number of subscribers to Internet services.

A public minded firm must send a message to every agent in the population, and we will call these agents recipients. The hypothesis that the message must be sent to all the recipients is a consequence of the fact that the social value of sending a message $V$ is larger than all the costs we consider. To make things as simple as possible, we will assume that $V=V_{s}+V_{r}$, where $V_{s}$ is the value to the sender of receiving a message and $V_{r}$ is the value to recipients of receiving it, and that both $V_{s}$ and $V_{r}$ are large. If messages are not sent through the Internet, they are sent through the postal service, where the cost to the sender is $\gamma$ per message. This cost is the total cost of sending the message, including not only postage, but also the cost of acquiring envelope and paper, printing the letter, stuffing the envelope, and so on. ${ }^{3}$

The Internet service which can be accessed through two technologies: a constant returns to scale (CRS) technology with a cost $c$ per message and an increasing returns to scale (IRS) technology with a fixed cost $F$ and a cost of 0 per consumer. We want to think about these costs in the following way. The sender is already connected to the Internet for other purposes, and the technologies are technologies of preparation of messages. With the CRS technology, messages are targeted one by one to their specific recipient. With the IRS technology, the preparation of Internet messages is directly linked to the databases of the organization. There would be basically no changes to the theory that follow if there were a modicum of returns to scale, with a small fixed cost, in the CRS technology ${ }^{4}$.

\footnotetext{
${ }^{3}$ In reality, there are probably increasing returns to scale in the sending of messages through the postal service. We assume that are primarily due to the fact that the sunk fixed costs are less than proportional to the number of recipients, and that these costs have already been sunk

${ }^{4}$ The theory could also be extended to the case where there are many technologies,
} 
The consumers have a type $v$ uniformly distributed on the segment ${ }^{5}[0,1]$, which represents there preferences for receiving Internet messages as opposed to postal messages. The consumer of type $v$ has a willingness to pay equal to $\tau+t v$ for receiving a Internet rather than a postal message. We assume that the constant $t$ is positive, but there are no restrictions on the sign of $\tau$.

We assume that the consumers of type $v \in[1-a, 1]$ are connected to the Internet, and we want to compute the welfare generated by the presence of the Internet as a function of $a$, that is as a function of the proportion of the population connected to the Internet. I make the hypothesis that it is the receivers with a high willingness to pay for Internet messages that are connected: this is reasonable, as they are the more likely to be willing to pay the cost of connection. This hypothesis is derived from more fundamental data in section 3 .

The sender maximizes social surplus, and we assume that the messages are sent efficiently to the "right" recipients. One simple hypothesis that ensures this is that the recipients are charged the relevant marginal cost, 0 , $\gamma$ or $c$ for receiving a message.

\subsection{Welfare with the CRS technology}

Let us first compute social welfare when the sender uses the CRS technology in order to send messages. The social benefit of sending an Internet message rather than a postal message to a recipient of type $v$ is $\tau+t v-c+\gamma$. Hence, recipients whose type $v$ satisfies

$$
v \geq \frac{c-\gamma-\tau}{t}
$$

will receive Internet messages while the others will receive postal messages. To make things interesting, we assume that inequality (1) is strictly satisfied for $v=1$, which means that the consumer with the higher type will receive an Internet message under the CRS technology:

$$
1>\frac{c-\gamma-\tau}{t}
$$

Let us first consider the case

$$
1-a \geq \frac{c-\gamma-\tau}{t}
$$

each indexed by a different pair of fixed and variable costs.

${ }^{5}$ There is no loss of generality in restricting $v$ to the segment $[0,1]$. Changes in the parameter $\tau$ defined below are equivalent to changes in the bounds of the interval. 
then recipients with $v \in[(c-\gamma-\tau) / t, 1-a]$ would like to receive E-mail messages, but are not connected. E-mail messages are sent to all connected recipients, whose types belong to the interval $[1-a, 1]$, and social welfare is

$$
V+\int_{1-a}^{1}(\tau+t v) d v-c a-\gamma(1-a)=(\tau+t-c+\gamma) a-\frac{t}{2} a^{2}-\gamma .
$$

On the other hand, when

$$
1-a \leq \frac{c-\gamma-\tau}{t}
$$

some connected recipients do not want to receive E-mail messages, and only recipients such that

$$
v \geq \frac{c-\gamma-\tau}{t}
$$

will receive them. Social welfare is

$$
\begin{aligned}
& V+\int_{\frac{c-\gamma-\tau}{t}}^{1}(\tau+t v) d v-c\left(1-\frac{c-\gamma-\tau}{t}\right)-\gamma\left(\frac{c-\gamma-\tau}{t}\right) \\
&=V+\tau-c+\frac{t}{2}+\frac{(c-\gamma-\tau)^{2}}{2 t} .
\end{aligned}
$$

The function that links social welfare to the proportion $a$ of the population that is connected is represented on figure 1 .

\subsection{Welfare with the IRS technology}

With the IRS technology, all connected recipients will be served with the Internet technology, and welfare will be

$$
V+\int_{1-a}^{1}(\tau+t v) d v-\gamma(1-a)-F=V+(\tau+t+\gamma) a-\frac{t}{2} a^{2}-\gamma-F
$$

if $a \leq 1+(\tau+\gamma) / t$. This is always satisfied if, as we will assume, $\tau+\gamma \geq 0$, that is if, once fixed costs are already sunk, it is efficient to send E-mails to the consumer of type 0 . This implies that the welfare with the IRS technology is increasing on $[0,1] .^{6}$ 


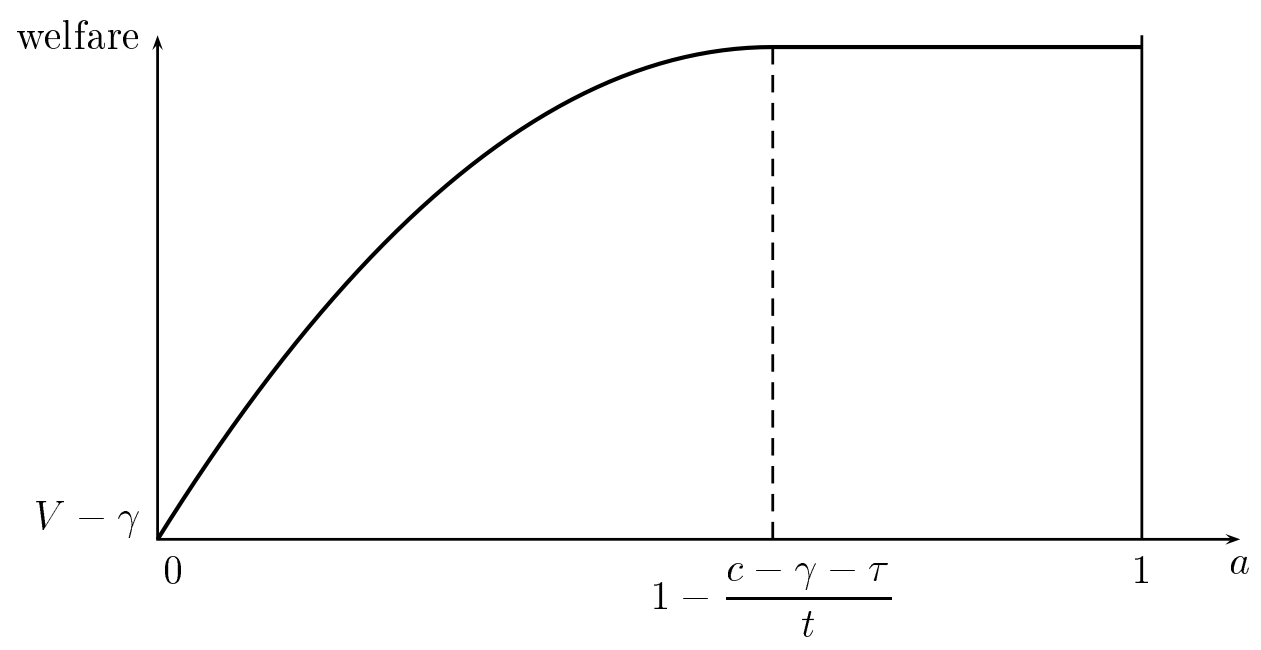

Figure 1: Social welfare as a function of $a$ with the CRS technology. Notice that the function is increasing for some values of $a$ and constant for large values, when the number of recipients who receive Internet messages is not increasing in $a$.

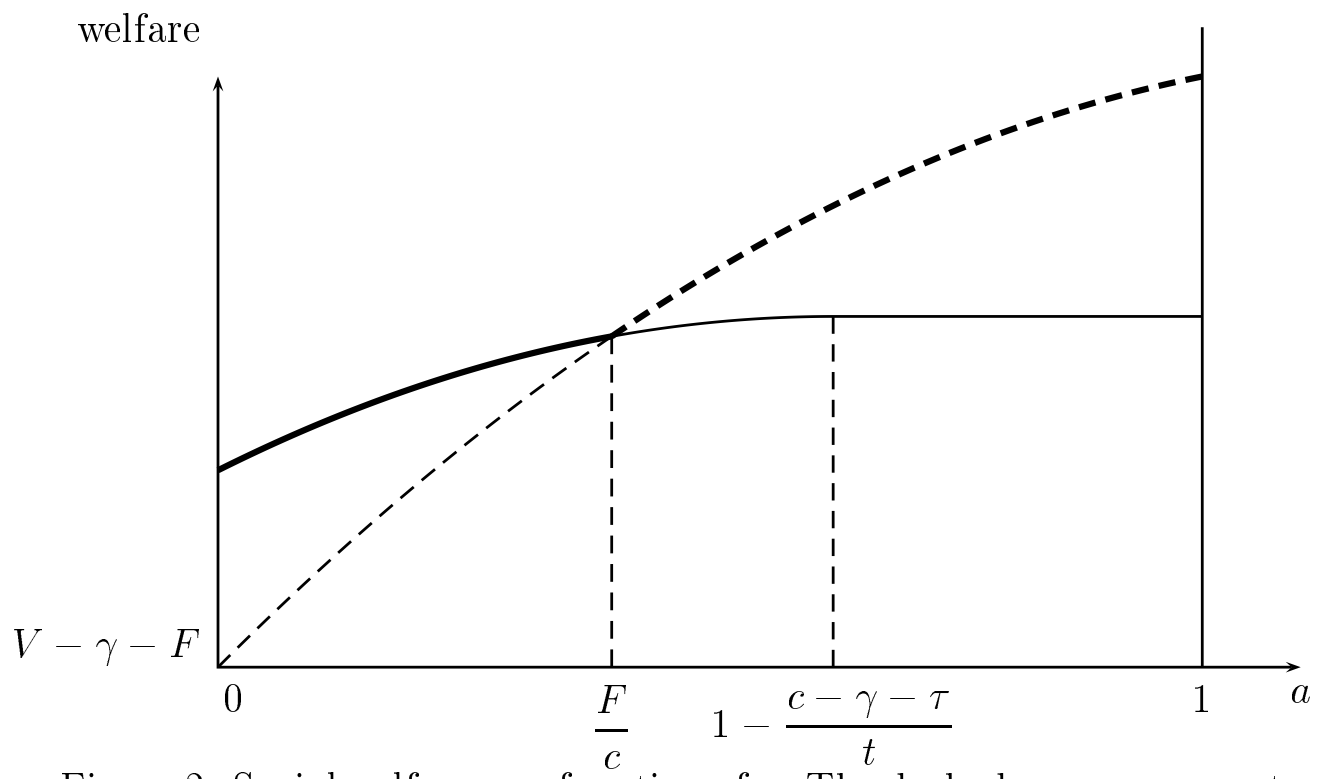

Figure 2: Social welfare as a function of $a$. The dashed curve represents social welfare with the IRS technology. The solid curve represents social welfare with the CRS technology. The bold curve represents social welfare with the optimal technology. 


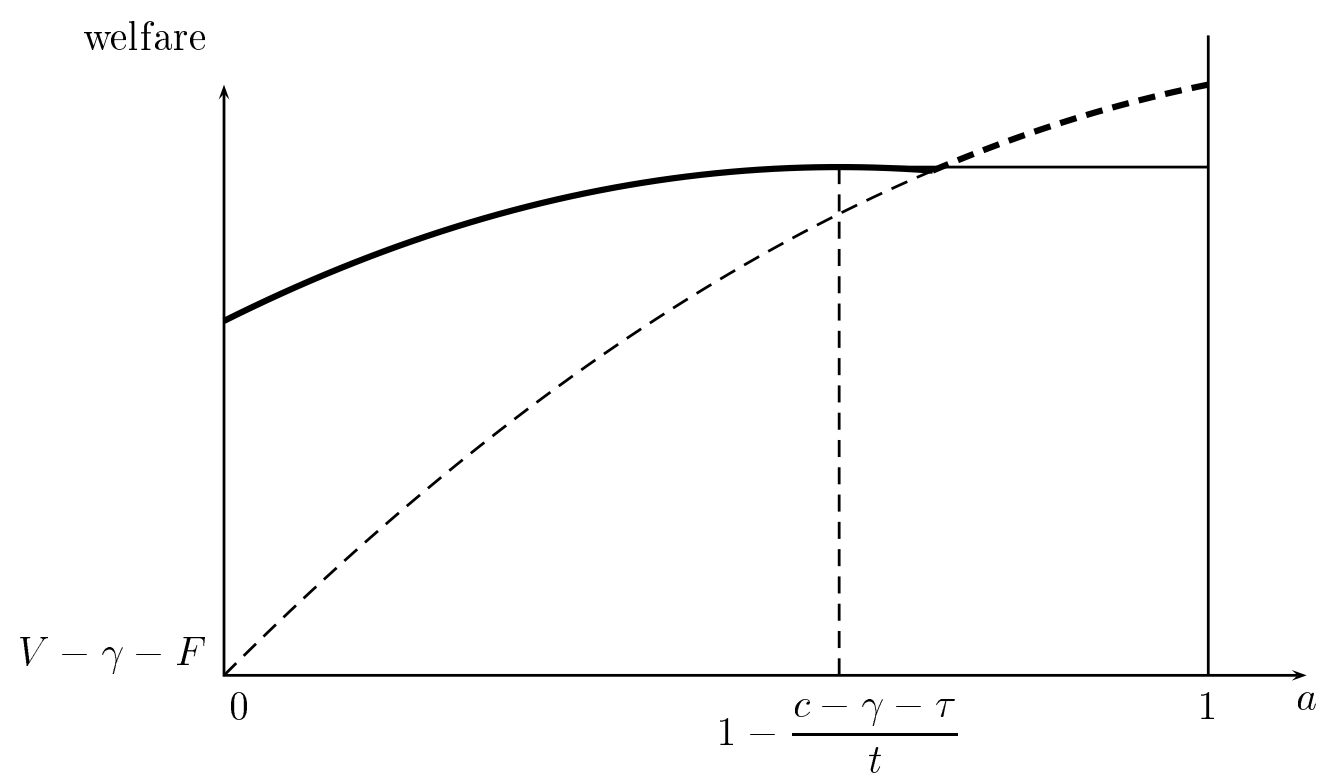

Figure 3: Social welfare as a function of $a$. The dashed curve represents social welfare with the IRS technology. The solid curve represents social welfare with the CRS technology. The bold curve represents social welfare with the optimal technology.

\subsection{Welfare as a function of $a$}

From the analysis that precedes, it is easy to compute welfare as a function of $a$, when the choice of the sender's technology is endogenous. Two cases need to be distinguished, which are represented on figures 2 and 3. In both cases the sender chooses the CRS technology for small as and the IRS technology for large $a$ s. Social welfare as a function of $a$ is the maximum of two concave functions. On figure 2, social welfare is strictly increasing in $a$, whereas on figure 3 it is constant for intermediate values of $a$.

For future reference, note that the flat portion of the social welfare curve for the CRS technology starts at

$$
a=\frac{t+\gamma+\tau-c}{t}
$$

whereas the intersection of the social welfare curves for the IRS and the CRS is at $a=F / c$ if this intersection is on the strictly increasing portion of the CRS curve. Figure 2 is therefore relevant when

$$
\frac{F}{c} \leq \frac{t+\gamma+\tau-c}{t}
$$

\footnotetext{
${ }^{6}$ Without any this assumption, the maximum welfare with the IRS technology would still obtained for a larger value of $a$ than with the CRS technology.
} 
In this case, the sender chooses the IRS technology when $a>F / c$ and the CRS technology when $a<F / c$.

\section{Coordination failures}

\subsection{The model}

The model developed above does not allow us easily to endogenize the decision of recipients to be connected to the Internet. It does not allow us either to discuss whether and how Internet access should be subsidized. In order to tackle these issues, we extend the model.

The recipients are described as above, but we will explicitly model the decision to connect or not to connect. In order to do so, assume that the cost of connection to the Internet is equal to $p$. This "price" should be interpreted as the difference between the price paid to the ISP, the Internet Service Provider which connects the recipient to the Internet, and the value of the services received from the Internet, other than receiving the messages on which this paper focuses. We assume that the ISP sector is competitive, so that this price is also equal to the social cost of connection.

There is a continuum of identical firms of mass 1. Each firm must send a message to all the recipients, and can be thought as an infinitesimal version of the sender described in section 2. As above, each firm has two possible techniques for sending these messages: a "postal technique" with constant returns to scale and cost $\gamma$ and an "Internet technology" with two possible technologies described as above. The firms are social welfare maximizers, but take their decisions in a decentralized fashion.

We will consider a two stage game. In the first stage, senders simultaneously decide on the technology that they will use and recipients decide whether to connect to the Internet; in the second stage, senders send messages.

In order to keep the paper manageably short and to facilitate the recycling of the results of section 2, we will only be interested in equilibria in which all the firms choose the same technology. This will be sufficient to point out the sources of coordination failures.

\subsection{Equilibria}

In order to ensure interior solutions, we will assume

$$
0<\frac{p-\gamma-\tau}{t}<1-\frac{c}{t}
$$


and in order to simplify computations, we will assume

$$
\frac{F}{c} \leq \frac{c-\gamma-\tau}{t}
$$

This implies that the function that links $a$ to social welfare has no flat part, as in figure 2 .

The second stage game is trivial: senders will send their messages efficiently to the recipients, and the welfare consequences of choices of technology and connection are the same as in section 2 .

In the first stage of the game, the senders and the recipients play a simultaneous move game, whose equilibrium is described by the following proposition.

\section{Proposition 1 If}

$$
\frac{F}{c}>1-\frac{p-\gamma-\tau}{t}
$$

there exists only one equilibrium, and in this equilibrium firms do not invest in the IRS technology.

If

$$
\frac{F}{c}<1-\frac{p-\gamma-\tau}{t}-\frac{c}{t}
$$

there exists only one equilibrium, and in this equilibrium firms invest in the IRS technology.

If

$$
1-\frac{p-\gamma-\tau}{t}-\frac{c}{t} \leq \frac{F}{c} \leq 1-\frac{p-\gamma-\tau}{t},
$$

there exist two equilibria, one in which firms invest in the IRS technology, and one in which they do not invest.

Proof. If a recipient of type $v$ believes that senders have invested in the IRS technology, his utility if he connects to the Internet is $-p+\tau+t v$ while his utility if he is not connected is $-\gamma$ (in order to simplify notation, I neglect the terms $V_{r}, V_{s}$ and $V$ in all the following expressions). He will connect if $-p+\tau+t v \geq-\gamma$, which is equivalent to $v \geq \frac{p-\gamma-\tau}{t}$, which by (4) belongs to $(0,1)$. Hence, if firms invest in the IRS technology $a=1-(p-\gamma-\tau) / t$.

If firms do not invest in the IRS technology, the recipient of type $v$ connects if $-p+\tau+t v-c \geq-\gamma$. Hence

$$
a=1-\frac{p-\gamma-\tau}{t}-\frac{c}{t}
$$

The welfare of senders is computed as above. By (5) firms will invest in the IRS technology if and only if $a \geq F / c$ (see section 2.4). 


\subsection{Welfare and governmental intervention}

Let us now turn to the computations of the welfare associated with these different equilibria. Notice first that given the choice of technology and the number of connected recipients, the outcome of the second stage of the game is efficient: Internet messages are sent to the right people.

In order to compute welfare, we need to correct the computations used in section 2 by subtracting the cost of connection $p a$. Therefore in an equilibrium in which firms invest in the IRS technology welfare will be

$$
(\tau+t+\gamma) a-\frac{t}{2} a^{2}-\gamma-F-p a=\frac{(t-p+\gamma+\tau)^{2}}{2 t}-\gamma-F
$$

as $a=1-(p-\gamma-\tau) / t$.

The computations are very slightly more complicated with the CRS technology, as we need to distinguish the cases where condition (3) is satisfied and the cases where it is not. It is satisfied if $a \leq(t-c+\gamma+\tau) / t$, which by (7) is equivalent to

$$
1-\frac{p-\gamma-\tau}{t}-\frac{c}{t} \leq \frac{t-c+\gamma+\tau}{t} \Longleftrightarrow p \geq 0,
$$

and we will assume in the sequel that these inequalities hold. Then, social welfare is equal to

$$
(\tau+t-c+\gamma) a-\frac{t}{2} a^{2}-\gamma-p a=\frac{(t-c-p+\gamma+\tau)^{2}}{2 t}-\gamma
$$

with $a$ computed through (7).

Coordination on the IRS technology is preferable to coordination on the CRS technology if

$$
\frac{(t-p+\gamma+\tau)^{2}}{2 t}-\gamma-F \geq \frac{(t-c-p+\gamma+\tau)^{2}}{2 t}-\gamma
$$

which is equivalent to

$$
\frac{F}{c} \leq 1-\frac{p-\gamma-\tau}{t}-\frac{c}{2 t}
$$

This implies that in the regions where there is only one equilibrium, the technology which will be chosen will indeed be the welfare maximizing technology. On the other hand, and not surprisingly, there can be coordination failure on the wrong equilibrium when several equilibria exist. When the optimal technology would be the CRS technology - that is for relatively 
large values of $F$ - the coordination failure implies too widespread an adoption of Internet technologies. When the optimal technology would be an IRS technology, the coordination failure implies too little adoption of Internet technology. It is in this latter case that some pump priming by the government could be warranted in order either to encourage the adoption of the IRS technology by the senders, or in order to make is less costly for recipients to connect to the Internet. In particular, it can subsidize the adoption of technology in such a way that the only equilibrium is the "good" equilibrium. In the model of this paper, the government could subsidize either the adoption of the IRS technology or the connection to Internet of the recipients. We will assume that it follows the following strategy: it gives a subsidy just sufficient to ensure that the only equilibrium is the equilibrium with adoption of the IRS technology. This is justified if there is a cost to public funds, as is commonly assumed in the new regulatory economics. Assume therefore that we have

$$
1-\frac{p-\gamma-\tau}{t}-\frac{c}{t}<\frac{F}{c}<1-\frac{p-\gamma-\tau}{t}-\frac{c}{2 t},
$$

and that the economy has settled on the CRS equilibrium, and let us define

$$
\delta=\frac{F}{c}-\left(1-\frac{p-\gamma-\tau}{t}-\frac{c}{t}\right) .
$$

If the government wants to subsidize the adoption of the IRS technology in such a way that the post subsidy fixed cost is equal to the right hand side of (6), it needs to provide a subsidy equal to $c \delta$. On the other hand, subsidizing the adoption of Internet by recipients (i.e., subsidizing $p$ ) requires a subsidy equal to $t \delta$. Inequality (4) imply $t \geq c$, which is furthermore reasonable: it is necessary and sufficient for the difference of valuation of an Internet message between a recipient with $v=1$ and a recipient with $v=0$ to be greater than the cost of sending that message with the CRS technology. Then, the optimal strategy of the government is to subsidize the adoption of the fixed cost technology by the senders, rather than the connection of recipients.

\section{References}

Gates, Bill with Hemingway, C. (1999), Business@ @he Speed of Thought: Using a Digital Nervous System, Penguin Books, London, New York.

Perkins, M. K. (1999), Text services and universal service obligations. mimeo, Howard University, Washington, DC. 